\title{
Transseptal direct complete annuloplasty: early experience
}

\author{
Jason H. Rogers ${ }^{1}$, Steven F. Bolling ${ }^{2}$ \\ ${ }^{1}$ Division of Cardiovascular Medicine, University of California, Davis Medical Center, Sacramento, CA, USA; ${ }^{2}$ Department of Cardiac Surgery, \\ University of Michigan Health System, Ann Arbor, MI, USA \\ Correspondence to: Jason H. Rogers, MD. Division of Cardiovascular Medicine, University of California, Davis Medical Center, Sacramento, CA \\ 95817, USA. Email: jhrogers@ucdavis.edu.
}

\begin{abstract}
Annuloplasty is a fundamental component of surgical mitral valve repair, and is employed in nearly $100 \%$ of repair operations for both primary and secondary mitral regurgitation (SMR). Developing transcatheter techniques to replicate surgical annuloplasty has been the focus of significant innovation and development in recent years. Since many patients are not offered surgery due to high perceived surgical risk, transcatheter approaches will provide new treatment options. In this manuscript, we review technologies which allow transseptal and transcatheter mitral valve (MV) annuloplasty.
\end{abstract}

Keywords: Mitral regurgitation; annuloplasty; transcatheter; transseptal

Submitted Mar 18, 2020. Accepted for publication Jun 09, 2020.

doi: 10.21037/acs-2020-mv-11

View this article at: http://dx.doi.org/10.21037/acs-2020-mv-11

\section{Introduction}

Since Carpentier first described "reconstructive valvuloplasty" in 1969 as a fundamental technique for mitral valve (MV) reconstruction, surgical mitral annuloplasty has become an integral part of successful surgical MV repair (1). For primary mitral regurgitation (PMR), annuloplasty is used adjunctively in nearly every surgical repair along with leaflet and chordal repair techniques. In a published survey from the Society of Thoracic Surgeons (STS) database of MV repair techniques for patients with degenerative disease undergoing operations between 2011 and 2016, annuloplasty was utilized in $96.1 \%$ of cases, leaflet resection in $58.9 \%$, and implantation of artificial expanded polytetrafluoroethylene (ePTFE) chords in $29.2 \%$ (2). In secondary mitral regurgitation (SMR), there are generally more prominent structural heart changes including left atrial (LA) and left ventricular (LV) enlargement, which lead to dilation of the mitral annulus. This dilation causes and contributes to MR progression in patients with SMR and LV dysfunction. By itself, mitral ring annuloplasty can correct SMR in patients who do not have excessive LV size or leaflet tethering.

Significant efforts have been made over the last two decades in an effort to replicate surgical mitral annuloplasty.
The eventual goal is to re-create a set of transcatheter techniques which can replicate the efficacy of surgical mitral valve repair with decreased morbidity. There are many patients that are not offered surgery due to high perceived surgical risk, and transcatheter approaches will provide new treatment options (3). In this manuscript, we review transcatheter annuloplasty techniques currently in development.

\section{Transcatheter annuloplasty}

Surgical mitral annuloplasty is performed on an open, arrested heart, where sutures can be placed under direct vision into the mitral annulus and trigones with great precision, avoiding delicate adjacent structures such as the mitral valve leaflets, coronary sinus (CS), and aortic leaflets. In chronic PMR, the goal of annuloplasty is to correct mitral annular enlargement and improve leaflet coaptation in patients who have developed a dilated mitral annulus. In patients with PMR and more normal annular dimensions, the rationale for annuloplasty is to increase durability of the repair by preventing subsequent mitral annular enlargement over time.

One of the greatest challenges of transcatheter mitral annuloplasty has been visualization and attachment of 
an annuloplasty device to the mitral annulus. The mitral annulus is a narrow strip of tissue, approximately $3-5 \mathrm{~mm}$ wide, that encircles the mitral annulus in a " $\mathrm{C}$ " shape which terminates at the anterior and posterior trigones. The intertrigonal segment (between the anterior and posterior trigones) is even a more challenging area for attachment since this consists of the aorto-mitral curtain, where the annular plane is essentially the point of continuity between the aortic root and the base of the anterior mitral leaflet. The mitral annulus is also mobile and moves with the cardiac cycle, although there is less movement in patients with LV dysfunction and SMR (4). Imaging for transcatheter annuloplasty also presents unique challenges related to anchor visualization, cardiac motion, and shadowing from the delivery system.

Initial devices designed for transcatheter annuloplasty were categorized as "indirect" annuloplasty, and attempted to leverage the proximity of the CS to the posterior mitral annulus. The basic mechanistic theory was that by modifying the shape of the CS, the adjacent posterior mitral annulus shape could be changed to improve mitral leaflet coaptation. Early studies with indirect annuloplasty showed a favorable effect on MR reduction, but since the mitral annulus and CS are not coplanar and are often at a significant distance from one another, mitral annular dimensions are more modestly affected. The circumflex coronary artery often courses between the CS and mitral annulus and it is at risk for compression, excluding patients from this approach (5).

More recently, there have been efforts at "direct" annuloplasty: replicating true surgical annuloplasty with attachment of either a ring or band directly to the mitral annulus using anchors under direct echocardiographic and fluoroscopic guidance. Challenges to this technique have been the need for extremely precise placement of the anchors on a moving heart, the risk of impinging adjacent structures, and anchor pull-out. The advantage of direct annuloplasty is that it directly influences the shape of the mitral annulus, most closely replicating surgical mitral annuloplasty. The current experience with various devices is summarized in the following section.

\section{Direct annuloplasty}

\section{Cardioband}

The Cardioband Mitral Valve Reconstruction System (Edwards Lifesciences, Irvine, California) is a transcatheter direct annuloplasty implant consisting of a flexible polymer sleeve with anchors that is delivered via a transfemoral and transseptal approach through a 25 French steerable sheath (Figure 1). This device has the most human implant experience of any direct transcatheter annuloplasty system and obtained Conformité Européene (CE Mark) approval in 2015. Patients are screened by echocardiography and computed tomography (CT). After performing transseptal puncture at the optimal location, 12 to 17 helical anchors are implanted through the Cardioband polyester sleeve at $8 \mathrm{~mm}$ increments onto the mitral annulus. The length of the device and total number of anchors implanted is determined by CT measurement of the mitral valve perimeter. The anchors are deployed initially at the lateral commissure/anterior trigone, working posteriorly and then up to the medial commissure/posterior trigone under fluoroscopic and transesophageal echocardiographic (TEE) guidance. Importantly, this device is not a complete ring annuloplasty but rather a partial band. After confirming optimal anchor placement and device positioning, a premounted contraction wire in the device connected to an adjusted spool is tensioned to achieve reduction in MR and the mitral annular size. Once optimal reduction is achieved, the delivery system is disconnected from the device.

Clinical results have been published initially for 31 patients treated with six month follow-up, and subsequently for 60 patients treated with one year follow-up. Nickenig et al. reported clinical results in 31 patients with symptomatic moderate to severe SMR and depressed LV function ( $\mathrm{LV}$ ejection fraction $34 \% \pm 11 \%$ ). Procedural success was $100 \%$ with no periprocedural deaths, and the mortality rate at 7 months was $9.7 \%$. The Cardioband device reduced the annular septolateral dimension from $3.7 \pm 0.5 \mathrm{~cm}$ at baseline to $2.4 \pm 0.4 \mathrm{~cm}$ at 6 months. The percentage of patients with FMR $\geq 3$ was reduced from baseline $77.4 \%$ to $13.6 \%$ at 65 months (of 31 patients treated, 22 had a six-month follow-up recorded in this report). There were also clinical improvements in New York Heart Association functional class, six-minute walk test, and other quality of life assessments (6). Messika-Zeitoun et al. reported on 60 patients with symptomatic moderate to severe SMR (mean LVEF $33 \% \pm 11 \%$ ). Of 60 patients treated, 39 were available for 12 month follow-up. This report highlighted some technical challenges with the device including two in-hospital deaths (not device-related), one stroke, two coronary artery complications and one tamponade. Anchor disengagement was observed in 10 patients, almost all of these occurring in the first 30 patients implanted prior to a device modification halfway through 


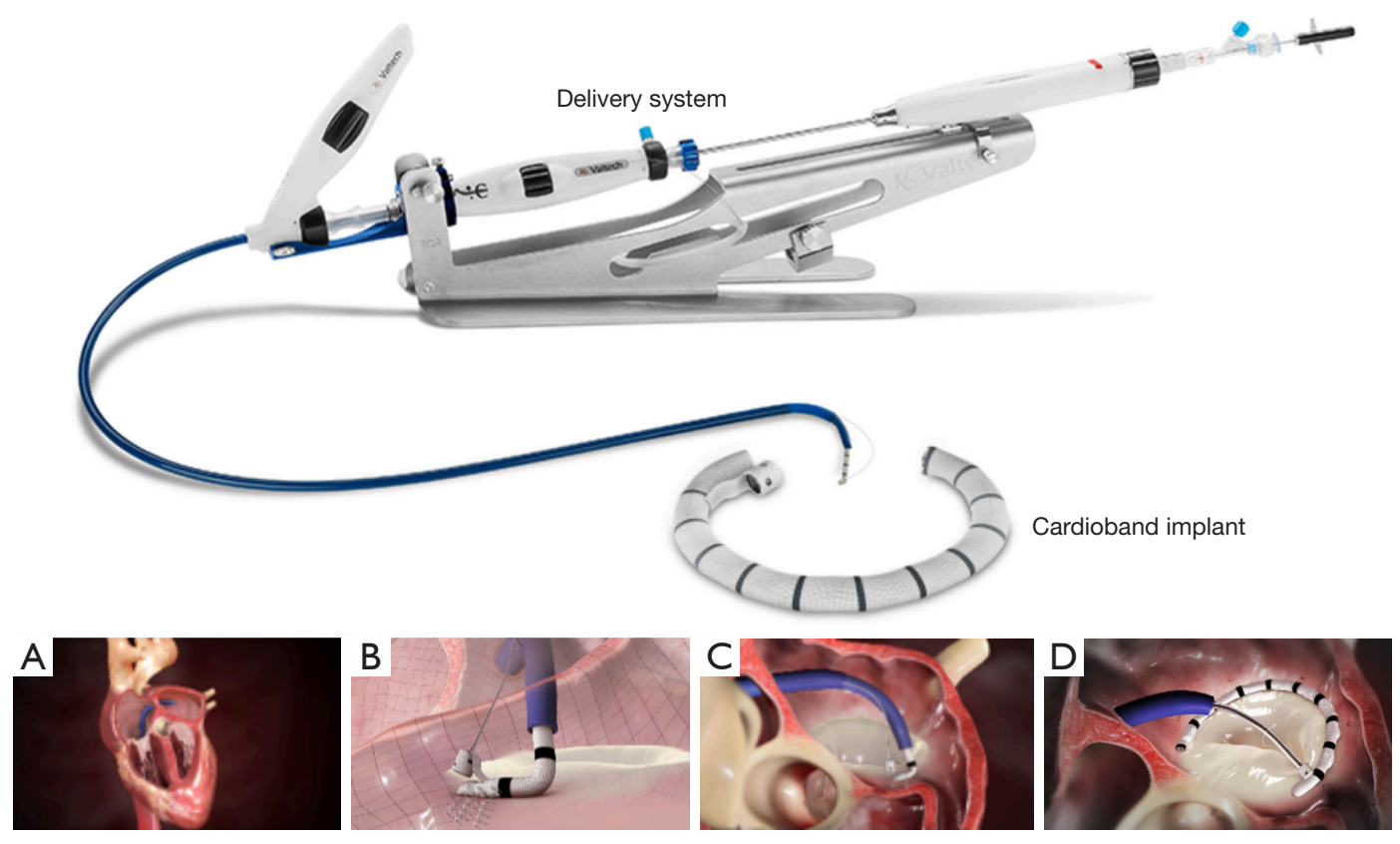

Figure 1 Cardioband direct annuloplasty. The Cardioband is delivered using a transseptal transfemoral delivery system (top panel). Steps of the procedure are (A) transseptal puncture in a predetermined optimal location by preprocedure CT scan, (B) initial helical anchor placement in the mitral annulus near the lateral commissure under fluoroscopic and TEE guidance, (C) continued implantation of anchors sequentially ending in region of medial commissure, and (D) final implant size adjustment and deployment. TEE, transesophageal echocardiography.

the study to mitigate the issue. At one year, overall survival, survival free of readmission for heart failure, and survival free of reintervention were $87 \%, 66 \%$ and $78 \%$ respectively. Mitral regurgitation at 12 months was moderate or less in $95 \%$ of the 39 patients who were available for follow-up and underwent transthoracic echocardiography at one year. Functional status and quality of life also improved overall (7).

The current status of the Cardioband mitral program most recently consists of the ACTIVE pivotal clinical trial (Annular ReduCtion for Transcatheter Treatment of Insufficient Mitral ValvE). This trial enrolls patients with clinically significant SMR who will be randomized 2:1 to receive either transcatheter mitral valve repair with the Edwards Cardioband System plus guideline directed medical therapy (GDMT), or GDMT alone. Patients will be seen for follow-up visits at discharge, 30 days, 6 months, and annually through 5 years. The primary outcome of the trial is the prevalence of $M R \leq 2+$ and a hierarchical comparison of device and control groups including time to cardiovascular death, number of heart failure hospitalizations, improvement in $6 \mathrm{MWT}$ distance and quality of life assessment. The current recruitment status of this trial is listed as "active, not recruiting" (clinicaltrials.gov NCT03016975).

\section{Millipede IRIS device}

The Millipede IRIS device (Boston Scientific, Maple Grove, Minnesota) is a semi-rigid, complete mitral annuloplasty ring made of nitinol with eight helical stainless-steel anchors pre-attached to the base of the ring. The device is delivered through a transfemoral transseptal sheath and steered down in a closed configuration to a position centered above the mitral annulus using TEE and fluoroscopic guidance. The device is then opened and advanced slowly until the helical anchor pads are contacting the mitral annulus. An integrated intracardiac echocardiography (ICE) catheter is delivered through the central lumen of the device and is used to clearly visualize each anchor and the target position on the mitral annulus without need for TEE (8). The advantage of the ICE catheter is that it is in the center of the device and therefore avoids shadowing from the delivery catheter, adjacent anchors, or other components of the device. Each anchor can be rotated independently driving 

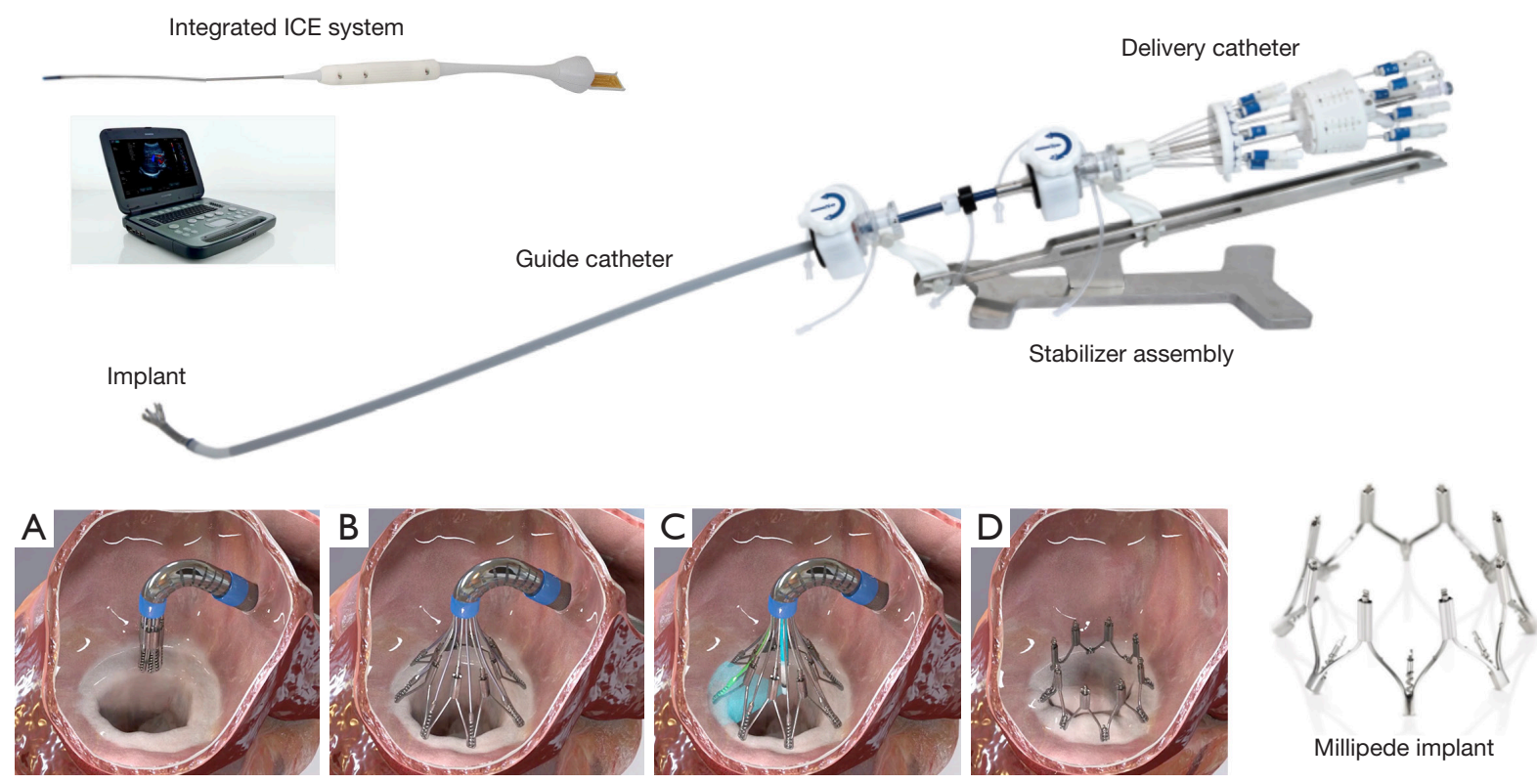

Figure 2 Millipede direct annuloplasty. The Millipede IRIS device consists of a nitinol frame, 8 helical anchors that attach to the mitral annulus, and 8 sliding collars that allow actuation of the device and reduction of the mitral annular diameter. There is an integrated intracardiac echocardiography (ICE) catheter which is placed through a central lumen in the delivery catheter to allow precise anchor visualization. Steps of the procedure are (A) standard transseptal puncture at a height of 4-5 cm above mitral annulus, after which the implant is steered down to the mitral valve in a closed configuration, (B) the ring is opened and advanced towards the mitral annulus, (C) integrated ICE catheter is used to place helical anchors in annulus, (D) ring after cinching and deployment.

that anchor securely into the mitral annulus. If the operator is not satisfied with initial placement, each anchor can be retracted or "unscrewed," moved, and redeployed. After all 8 helical anchors are attached to the mitral annulus, the device can be actuated to reduce the mitral annular diameter. This is achieved through 8 sliding collars which can be manipulated individually, drawing the two adjacent helical anchors closer together. Because each collar can be individually tensioned, operators can customize the final annular circumference and diameter. The device can be repositioned and retrieved up until the ring is fully released from the delivery system. In summary, the IRIS procedure consists of three basic steps: (I) placement, (II) anchoring, and (III) actuation (Figure 2).

Rogers et al. reported the first-in-human experience with the Millipede device in 2018 (9). Patients in this study had symptomatic 3 or 4+ ischemic or nonischemic MR with a dilated mitral annulus and the average LVEF was $42 \% \pm 19 \%$. Mitral annular dimensions were measured by transthoracic and transesophageal echocardiography and CT. MR quantification was performed using the Mitral Valve Academic Research Consortium (MVARC) criteria (10). The study consisted of phase 1, during which the device was implanted surgically under direct vision to establish the safety and efficacy of the implant, and phase 2, where a lower-profile transcatheter IRIS ring was delivered to the mitral annulus using a transfemoral, transseptal approach.

Seven patients received the IRIS ring in phase $1(n=4)$ and phase $2(n=3)$. There was no device-related procedural death, stroke, or MI. In all of the patients who received the IRIS device, the pre-procedure mitral SL diameter as determined by TTE was reduced from a baseline of $38.0 \pm 4.1 \mathrm{~mm}$ to $25.9 \pm 4.9 \mathrm{~mm}$ at 30 days. Every patient in the study demonstrated reduction of MR, with all patients showing a decline from a baseline of 3 or $4+$ MR to 0 or $1+M R$ at 30 days. There were also improvements in NYHA Class, LV diastolic volumes, and LA volumes. A current generation Millipede transcatheter ring is currently undergoing clinical evaluation in human clinical trials.

\section{Valcare AMEND}

The AMEND system (Valcare, Israel) is a rigid mitral 

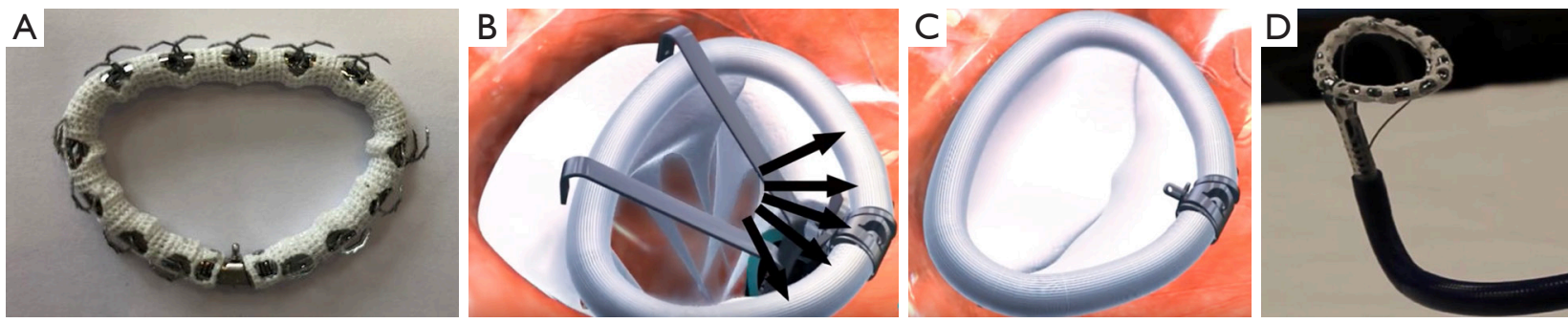

Figure 3 AMEND direct annuloplasty. (A) The AMEND annuloplasty ring; (B) the device can be delivered through a transapical sheath and initially anchored to the posterior annulus (arrows); (C) final device appearance after pulling and anchoring anteriorly, followed by separation from delivery catheter; (D) transseptal delivery system in development.

annuloplasty ring that can be directly attached to the mitral annulus using either a transapical or transfemoral transseptal system (Figure 3). The device has a unique design in that it is delivered in a linear manner through a catheter, but can reform into a rigid " $\mathrm{D}$ " shaped ring once in the left atrium. The device has a series of barbed anchors that can be activated first to allow anchoring on the posterior annulus. The device is then pulled and anchored anteriorly, followed by separation from the delivery catheter and deployment. Although there is currently no published data, the company reports successful human implantation of the ring using a transapical delivery system. A transfemoral transseptal delivery system is in development, as well as a docking transcatheter valve-in-ring system.

\section{Indirect annuloplasty}

\section{Carillon device}

The Carillon device (Cardiac Dimensions, Kirkland, Washington) is delivered to the CS through a 9 French right internal jugular vein guide. The device is made of nitinol and, initially, a distal anchor that is deployed deeply in the CS. Next, backwards traction is applied, followed by deployment of the proximal anchor which cinches the posterior peri-annular tissue, reducing mitral annular dimensions (Figure 4). To date there have been over 1,000 patients treated with this device and it received CE Mark approval in 2011. Single arm trials including the TITAN and TITAN II trials showed that in patients with functional $M R$, the device reduced MR, generated favorable LV remodeling, and reduced heart failure hospitalizations $(11,12)$. The randomized REDUCE-FMR (Carillon Mitral Contour System for Reducing Functional Mitral Regurgitation) was a sham control randomized trial of 120 patients with secondary MR all receiving optimal medical therapy, randomized to a sham procedure or the Carillon device. The mean $L V$ ejection fraction was $34 \% \pm 11 \%$. At 12 months the primary endpoint was met, with a mean reduction of mitral regurgitant volume by $7.1 \mathrm{~mL} /$ beat in the treatment group compared to an increase of $3.3 \mathrm{~mL} / \mathrm{beat}$ in the sham group. There were also significant reductions in $\mathrm{LV}$ volumes in the treatment arm. These encouraging results have led to the ongoing CARILLON prospective, multi-center, randomized, double-blind pivotal trial to assess the safety and efficacy of the CARILLON Mitral Contour System in treating subjects with functional MR associated with heart failure, compared to a randomized control group which is medically managed according to heart failure guidelines. The primary safety endpoint is freedom from a composite of major adverse events in the intervention group greater than a performance goal of $90 \%$. The primary efficacy objective is to demonstrate that the intervention group is superior to the control group on the hierarchical composite endpoint of death, transplant or LVAD, percutaneous or surgical mitral valve intervention, heart failure hospitalization, and improvement in sixminute walk distance at 12 months (clinicaltrials.gov NCT03142152).

\section{ARTO device}

The ARTO device (MVRx Inc., Belmont, California) is comprised of a CS anchor (T-bar) and an interatrial septal anchor connected by a suture, the length of which can be adjusted to reduce the antero-posterior diameter of the mitral annulus. The device is delivered using mostly fluoroscopic guidance but also requires TEE. First, the CS T-bar is placed from a right internal jugular venous approach. Next, transseptal puncture is performed, and by use of magnetic catheters, a suture is passed safely from the 

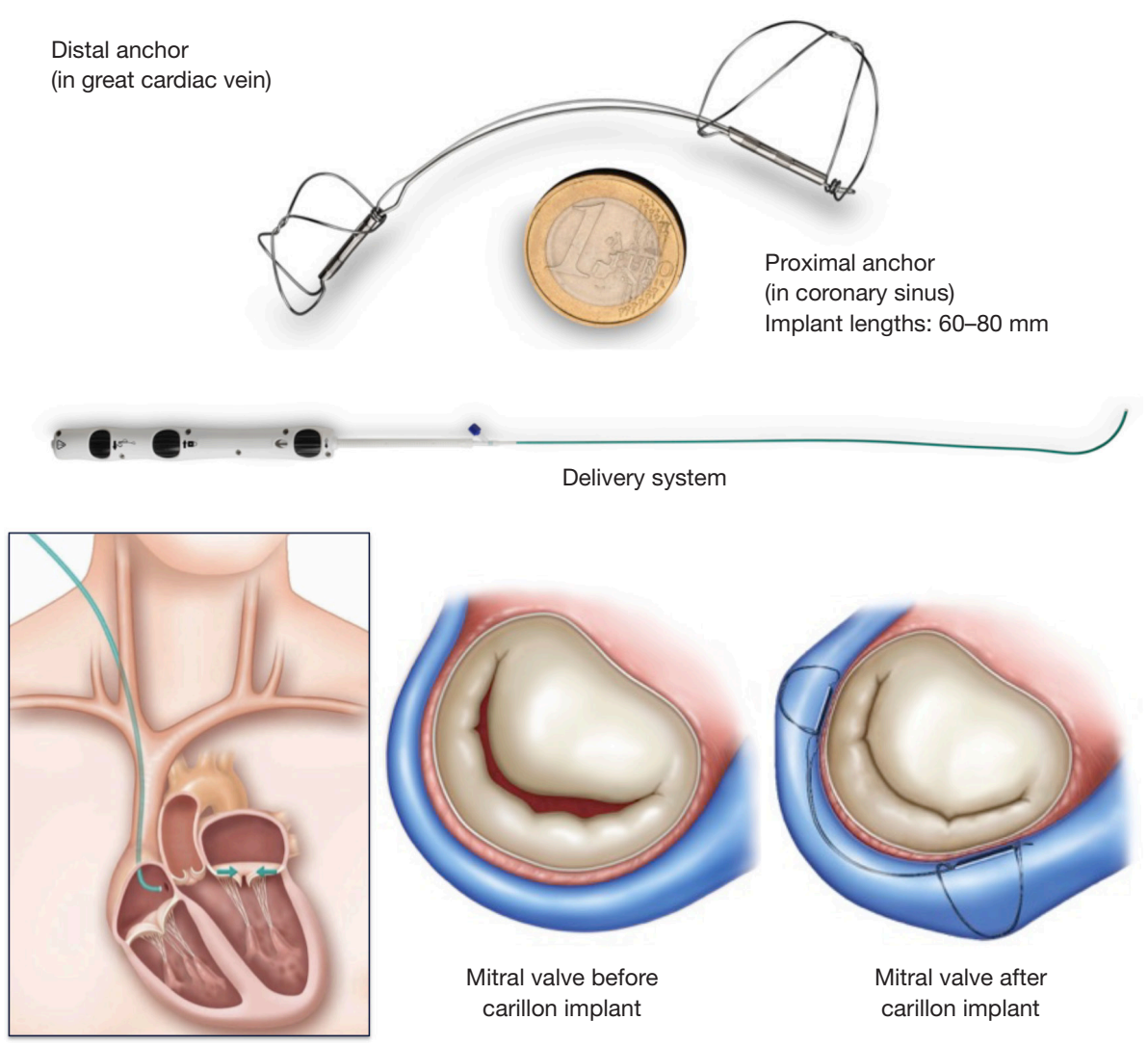

Figure 4 Carillon indirect annuloplasty. The Carillon device comes in lengths $60-80 \mathrm{~mm}$ and anchor sizes to fit a variety of coronary sinus anatomies. The device is delivered from the right internal jugular vein and improves mitral leaflet coaptation by cinching the coronary sinus.

CS into the left atrium. This suture is then externalized through the transseptal puncture site to the femoral vein, over which an Amplatzer-style atrial septal anchor is placed. The suture is tensioned to the desired degree, locked, and cut (Figure 5). The MAVERIC (MitrAl ValvE RepaIr Clinical) trial enrolled 11 patients at a single center with SMR treated with the ARTO system. At 2 year follow-up, there were continued significant improvements compared to baseline in MR grade, regurgitant volumes, and reduced mitral annular antero-posterior diameter (13).

\section{Combination therapy}

An intriguing application of transcatheter annuloplasty is in combining this therapy with other transcatheter leaflet repair systems to treat more complex disease. There are numerous case reports describing combined therapy, but studying this approach on a larger scale becomes challenging due to the need to combine investigational therapies, reimbursement issues, and standardizing the technical approach $(14,15)$. There is significant clinical experience with valve in ring therapy for transcatheter mitral valve replacement (TMVR). It is likely that transcatheter mitral annuloplasty devices will serve as "docking stations" for TMVR devices in the future and these concepts are currently in development.

\section{Discussion}

Mitral valve annuloplasty is employed in almost every surgical mitral valve repair and is an essential part of the "gold standard" surgical technique. For patients with SMR, restrictive annuloplasty can be used to normalize the anterior-posterior mitral annular diameter as a stand-alone therapy. In patients with PMR, annuloplasty rings have been used to downsize or stabilize the annulus as an adjunct to leaflet/chordal repair. The benefits and challenges of transcatheter mitral annuloplasty are summarized in Table 1.

Although transcatheter annuloplasty is in relative infancy compared to its longstanding surgical counterpart, we can 

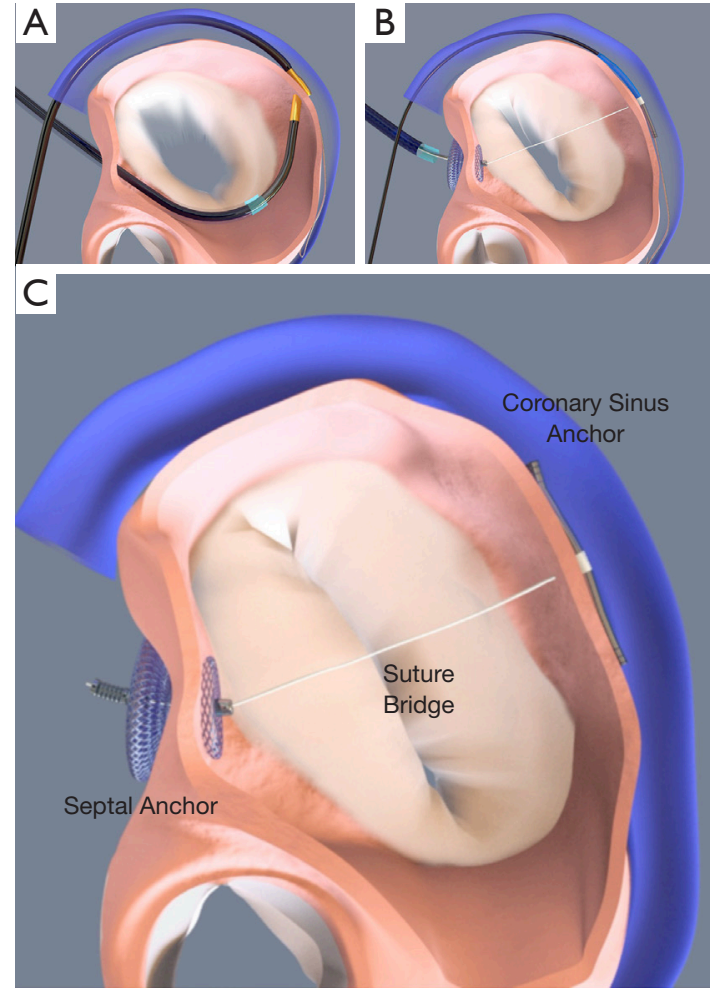

Figure 5 ARTO indirect annuloplasty. (A) The procedure is performed with transfemoral transseptal and right internal jugular vein access. Magnetic catheters are used to direct a wire safely from the CS to the left atrium; (B) ARTO device with CS and septal anchors in place before tensioning of suture bridge. At this point the device is fully retrievable; (C) final appearance after tensioning the system, locking, and cutting the free end of the suture in the right atrium. CS, coronary sinus.

learn important lessons from this deep surgical experience. There is evidence that complete (closed) rigid annuloplasty rings have less MR recurrence over time than partial (open) flexible bands. Spoor et al. retrospectively reviewed outcomes in 289 patients with $\mathrm{LVEF} \leq 30 \%$ who received an undersized complete mitral annuloplasty ring for MV repair in the setting of ischemic or idiopathic cardiomyopathy. One hundred and seventy patients had a flexible complete ring and 119 patients received a 26 or $28 \mathrm{~mm}$ undersized nonflexible complete ring. In the flexible group, 9.4\% required a repeat procedure because of significant recurrent MR (average time to reoperation 2.4 yrs), compared with only $2.5 \%$ in the nonflexible group (average time to reoperation $4.0 \mathrm{yrs}$ ) (16).

Multiple quantitative echocardiographic measurements have also been studied and may predict patients who respond more favorably to stand-alone annuloplasty. Braun et al. studied 100 patients with ischemic MR undergoing restrictive mitral annuloplasty (median size $26 \mathrm{~mm}$ ) and CABG. In this study, a preoperative LV enddiastolic diameter (LVEDD) $>65 \mathrm{~mm}$ predicted worse survival $(80 \% \pm 5.2 \%$ vs. $49 \% \pm 11 \%$ at 5 years) (17). Magne et al. reported that in 51 patients undergoing restrictive annuloplasty for ischemic MR, a posterior leaflet angle $\geq 45$ degrees predicted postoperative persistence of MR (18). Increased leaflet tethering into the left ventricle has also been quantified by Calafiore $e t$ al. who examined 49 patients with mixed ischemic and nonischemic cardiomyopathy, who underwent either flexible annuloplasty repair $(n=29)$ or replacement $(n=20)$. The mean postoperative $M R$ grade was higher when preoperative MV coaptation depth was $\geq 11$ $(2.5 \pm 0.7) \mathrm{mm}$ as compared to $<11(1.3 \pm 0.8) \mathrm{mm}(19)$.

There remains an ongoing debate as to when MV replacement may be preferable to repair in the management of ischemic (secondary) MR. Acker et al. randomized 251 patients with ischemic MR 1:1 to CABG + MV replacement vs. CABG + MV repair. The primary end point was the LV end-systolic volume index (LVESVI) at 1 year. Although there was no difference in death at 12 months $(17.6 \%$ vs. $14.3 \%, \mathrm{P}=\mathrm{NS}$ replace $v s$. repair) or LVESVI $\left(60.7 \pm 31.5\right.$ vs. $54.6 \pm 25.0 \mathrm{~mL} / \mathrm{m}^{2}, \mathrm{P}=\mathrm{NS}$ repair $v s$. replace), recurrent moderate or severe $\mathrm{MR}$ was higher with repair than replacement $(32.6 \%$ vs. $2.3 \%, \mathrm{P}<0.001)$. Importantly, at 1 year, patients in the repair group without recurrent MR demonstrated a more favorable decrease in LVESVI $\left(47.3 \pm 23.0 \mathrm{~mL} / \mathrm{m}^{2}\right)$ than both patients with repair and recurrent MR and patients with replacement (20). A subsequent analysis by Kron $e$ al. of 116 patients who were randomized to the mitral repair group sought to identify patients that would benefit most from mitral annuloplasty. They identified that the degree of mitral valve leaflet tethering was a powerful predictor of recurrent MR, and that in particular the presence of basal aneurysm/dyskinesis (found in 52/116 patients undergoing repair) was strongly associated with recurrent MR. We can conclude from this data that properly selected patients for mitral annuloplasty who have successful repair have superior outcomes in terms of LV size and remodelling compared to those patients undergoing replacement (21).

\section{Conclusions}

Transcatheter mitral annuloplasty holds great promise as 


\begin{tabular}{ll} 
Table 1 Transcatheter mitral annuloplasty & \\
\hline Pros & Cons \\
\hline Long history of surgical experience and efficacy & Technical and imaging challenges still being evaluated \\
\hline Used in both primary and secondary MR repair & $\begin{array}{l}\text { Anatomic exclusions for annuloplasty (excessive leaflet } \\
\text { tethering, annular dilation or left ventricular dilation) }\end{array}$ \\
\hline No leaflet or LVOT interference & Does not fully address mixed etiology (prolapse, flail, clefts) \\
\hline Minimal risk of mitral stenosis & Long term durability unknown \\
\hline Can be used with chordal/leaflet technologies & \\
\hline Can serve as a docking station for transcatheter mitral valve replacement & \\
\hline LVOT, left ventricular outflow tract. & \\
\hline
\end{tabular}

an effective technique to reduce mitral annular dimensions and improve leaflet coaptation, mostly in patients with secondary MR, while leaving numerous options open for future therapies. Ongoing improvements in catheter design and improved imaging will make these procedures adoptable to a wide spectrum of operators.

\section{Acknowledgments}

Funding: None.

\section{Footnote}

Conflicts of Interest: Drs. Bolling and Rogers consultants to Abbott, Boston Scientific, and Pipeline Medical Technologies, a wholly-owned subsidiary of W. L. Gore \& Associates. Inc.

Open Access Statement: This is an Open Access article distributed in accordance with the Creative Commons Attribution-NonCommercial-NoDerivs 4.0 International License (CC BY-NC-ND 4.0), which permits the noncommercial replication and distribution of the article with the strict proviso that no changes or edits are made and the original work is properly cited (including links to both the formal publication through the relevant DOI and the license). See: https://creativecommons.org/licenses/by-nc-nd/4.0/.

\section{References}

1. Carpentier A. Reconstructive valvuloplasty. A new technique of mitral valvuloplasty. Presse Med 1969;77:251-3.
2. Gammie JS, Chikwe J, Badhwar V, et al. Isolated Mitral Valve Surgery: The Society of Thoracic Surgeons Adult Cardiac Surgery Database Analysis. Ann Thorac Surg 2018;106:716-27.

3. Iung B, Baron G, Butchart EG, et al. A prospective survey of patients with valvular heart disease in Europe: The Euro Heart Survey on Valvular Heart Disease. Eur Heart J 2003;24:1231-43.

4. Matos J, Kronzon I, Panagopoulos G, et al. Mitral annular plane systolic excursion as a surrogate for left ventricular ejection fraction. J Am Soc Echocardiogr 2012;25:969-74.

5. Tops LF, Van de Veire NR, Schuijf JD, et al. Noninvasive evaluation of coronary sinus anatomy and its relation to the mitral valve annulus: implications for percutaneous mitral annuloplasty. Circulation 2007;115:1426-32.

6. Nickenig G, Hammerstingl C, Schueler R, et al. Transcatheter Mitral Annuloplasty in Chronic Functional Mitral Regurgitation: 6-Month Results With the Cardioband Percutaneous Mitral Repair System. JACC Cardiovasc Interv 2016;9:2039-47.

7. Messika-Zeitoun D, Nickenig G, Latib A, et al. Transcatheter mitral valve repair for functional mitral regurgitation using the Cardioband system: 1 year outcomes. Eur Heart J 2019;40:466-72.

8. Rogers JH, Boyd WD, Smith TW, et al. Novel IntraAnnular Mitral Valve Imaging With Intracardiac Echocardiography for Direct Transcatheter Mitral Annuloplasty. JACC Cardiovasc Interv 2019;12:2443-5.

9. Rogers JH, Boyd WD, Smith TW, et al. Transcatheter Annuloplasty for Mitral Regurgitation with an Adjustable Semi-Rigid Complete Ring: Initial Experience with the Millipede IRIS Device. Structural Heart 2018;2:43-50.

10. Stone GW, Vahanian AS, Adams DH, et al. Clinical 
Trial Design Principles and Endpoint Definitions for Transcatheter Mitral Valve Repair and Replacement: Part 1: Clinical Trial Design Principles: A Consensus Document From the Mitral Valve Academic Research Consortium. J Am Coll Cardiol 2015;66:278-307.

11. Siminiak T, Wu JC, Haude M, et al. Treatment of functional mitral regurgitation by percutaneous annuloplasty: results of the TITAN Trial. Eur J Heart Fail 2012;14:931-8.

12. Lipiecki J, Siminiak T, Sievert H, et al. Coronary sinusbased percutaneous annuloplasty as treatment for functional mitral regurgitation: the TITAN II trial. Open Heart 2016;3:e000411.

13. Erglis A, Narbute I, Poupineau M, et al. Treatment of Secondary Mitral Regurgitation in Chronic Heart Failure. J Am Coll Cardiol 2017;70:2834-5.

14. Ristalli F, Meucci F, Stolcova M, et al. MitraClip Implantation to Treat Early Recurrence of Mitral Regurgitation After Percutaneous Direct Annuloplasty With Cardioband. JACC Cardiovasc Interv 2018;11:1416-7.

15. Rogers JH, Boyd WD, Smith TWR, et al. Combined MitraClip Edge-to-Edge Repair With Millipede IRIS Mitral Annuloplasty. JACC Cardiovasc Interv

Cite this article as: Rogers JH, Bolling SF. Transseptal direct complete annuloplasty: early experience. Ann Cardiothorac Surg 2021;10(1):57-65. doi: 10.21037/acs-2020-mv-11
2018;11:323-4.

16. Spoor MT, Geltz A, Bolling SF. Flexible versus nonflexible mitral valve rings for congestive heart failure: differential durability of repair. Circulation 2006;114:I67-71.

17. Braun J, van de Veire NR, Klautz RJ, et al. Restrictive mitral annuloplasty cures ischemic mitral regurgitation and heart failure. Ann Thorac Surg 2008;85:430-6; discussion 436-7.

18. Magne J, Pibarot P, Dagenais F, et al. Preoperative posterior leaflet angle accurately predicts outcome after restrictive mitral valve annuloplasty for ischemic mitral regurgitation. Circulation 2007;115:782-91.

19. Calafiore AM, Gallina S, Di Mauro M, et al. Mitral valve procedure in dilated cardiomyopathy: repair or replacement? Ann Thorac Surg 2001;71:1146-52; discussion 1152-3.

20. Acker MA, Parides MK, Perrault LP, et al. Mitral-valve repair versus replacement for severe ischemic mitral regurgitation. N Engl J Med 2014;370:23-32.

21. Kron IL, Hung J, Overbey JR, et al. Predicting recurrent mitral regurgitation after mitral valve repair for severe ischemic mitral regurgitation. J Thorac Cardiovasc Surg 2015;149:752-61.e1. 